\title{
RATIONALITÉ CONTEXTUELLE ET PRÉSUPPOSÉ COGNITIF LE CAS LOMBROSO
}

\author{
Marc RenNeVILle
}

RÉsumÉ : Il est proposé une réflexion épistémologique sur la posture que l'historien des sciences humaines peut adopter vis-à-vis de ses objets d'investigation. La théorie du "criminel-né » de Cesare Lombroso, produite dans le dernier tiers du $\mathrm{XIX}^{\mathrm{e}}$ siècle est prise ici comme exemple et support pour la discussion parce qu'elle apparaît comme un cas limite, presque caricatural, tant par sa formulation initiale que par son traitement dans la mémoire disciplinaire. Les approches normatives ou «présentistes » ne pouvant rendre compte de la logique du discours de Lombroso que sur le mode de la dénégation, il a semblé préférable - sans qu'il soit nécessaire de devenir lombrosien - de suivre un autre fil directeur. On suggère de substituer à une posture épistémologique présentiste les concepts de "rationalité contextuelle " et de " présupposé cognitif ». Ces concepts, combinés à une plus grande sensibilité aux différentes durées pourraient initier une approche qui serait à la fois plus spécifiquement historique et ouverte aux apports des autres sciences humaines.

Mots-CLÉs : criminologie, historiographie, Lombroso, rationalité, temporalités.

ABSTRACT : This article proposes an epistemological reflexion about the posture of the historian of human sciences towards his objects. Cesare Lombroso's theory of criminal anthropology was taken here as an example of the discussion because it seemed to be a caricatural case. Considering that normative or "whiggish " perspectives cannot account for the logic of Lombroso's discourse, we attempt to develop here an alternative posture from which extrapolations could be made to other fields. This alternate is found in the introduction of the concepts of "contextual rationality " and "cognitive presupposition » and in a reconsideration of the time duration.

KEYWORDS : criminology, historiography, Lombroso, rationality, temporalities.

Revue de synthèse : $4^{e}$ S. $\mathrm{N}^{0}$ 4, oct.-déc. 1997, p. 495-529. 
Zusammenfassung : Der Artikel fragt nach dem epistemologischen Standort des Historikers der Humanwissenschaften. Als Beispiel wird dafür Cesare Lombrosos Theorie des " geborenen Verbrechers " aus der zweiten Hälfte des 10. Jahrhunderts herangezogen, die sich sowohl aufgrund ihrer Formulierung wie aufgrund ihrer Rezeption innerhalb der Disziplin anbietet. Da dem normativen oder gegenwartsbezogenen Ansatz die spezifische Logik des Diskurses Lombrosos verschlossen bleibt, schien es sinnvoll - ohne deswegen Lombrosos Thesen zu folgen - ein anderes Argumentationsmuster zu wählen. Es wird vorgeschlagen, anstelle des präsentistischen Zugangs Begriffe wie den einer "kontextgebundenen Rationalität " bzw. der « kognitiven Vorgabe » zu wählen. Beide Begriffe scheinen geeignet durch eine grössere Sensibilität gegenüber den unterschiedlichen temporalen Vorgegebenheiten einen spezifisch historischen bzw. geisteswissenschaftlichen Zugang zu erleichtern.

STICHWÖRTER : Kriminalwissenschaft, Geschichtsschreiber, Lombroso, Rationalität.

Marc RenNevile, né en 1967, titulaire d'un doctorat d'histoire portant sur La Médecine du crime, 1785-1885 (Villeneuve-d'Ascq, Presses universitaires du Septentrion, 1997), est chercheur associé à l'Institut d'histoire de la médecine de l'université Paris 5 . Ses recherches actuelles portent sur le mouvement phrénologique français et la médicalisation des déviances.

Adresse : 1, place Winston-Churchill, 95300 Pontoise.

Courrier électronique : renneville@paris7.jussieu.fr 
« Face à l'“anormalité" des crimes qu'on lui attribuait, la "normalité" de l'accusé, tant physique que morale, posait au tribunal le problème de la responsabilité."

Leonardo SCIASCIA, « Le procès », in La Mer couleur de vin, Paris, Gallimard, 1990, p. 185-186.

L'histoire des sciences de l'homme ne cesse en France d'appréhender de nouveaux objets et l'ouvrage récemment consacré à la criminologie française en est un bon témoin ${ }^{1}$. Dans ce livre, et plus encore dans le colloque qui l'avait précédé, ce fut paradoxalement le médecin italien Cesare Lombroso (1835-1909) qui tint la vedette. Il est vrai que la renommée de ce savant dépasse très largement le cénacle des historiens avertis et que la notion de «criminel-né » garde une place importante dans la mémoire disciplinaire des criminologues contemporains. Cette dernière s'articule sur l'idée directrice que la théorie de Lombroso eut une diffusion inversement proportionnelle à sa valeur scientifique et que, sous ce rapport, elle ne fut qu'un tissu d'erreurs mal ficelées, voire, selon Pierre Darmon, une « mystification pure et simple » ou, selon Jean-Michel Labadie, une " trouvaille théorique » destinée à « sombrer lamentablement dans l'oubli » ${ }^{2}$. Ce jugement rétrospectif est partagé par bon nombre d'historiens et de criminologues qui désignent à la fois Lombroso comme le précurseur de la criminologie scientifique et l'exemple même de ce qu'il ne faut pas faire. Lombroso exhibant des monstres est ainsi devenu à son tour un monstre épistémologique à l'aune de la vraie criminologie comme de l'histoire de la vérité. Si le renversement de perspective est net, la présence continue d'une dénégation ne l'est pas moins, chez Lombroso comme chez les historiens.

Il est possible d'éviter ce redoublement de la dénégation de Lombroso en suspendant à des fins méthodologiques notre approche normative en terme de "vérité ». Toutefois, le récit produit par l'historien des discours scientifiques ne pourra se démarquer nettement de l'évaluation rétrospective du criminologue contemporain qu'à condition de mener une réflexion épistémologique sur les catégories qui façonnent son récit : celle de «temps » bien sûr, mais aussi celle de «théorie » et de « rationalité ». Une perspective réflexive incite en particulier à rendre problématique l'usage du

1. [Pour plus de précisions sur les références citées en notes, se reporter à la bibliographie finale, p. 525-529.] Ici, Mucchielli, éd., 1995.

2. Darmon, 1989, p. 104; Labadie, 1995, p. 323. 
concept de "rationalité » dans l'histoire des sciences humaines. C'est ce qui sera suggéré ici en rappelant, dans un premier temps, les grandes lignes de l'anthropologie criminelle de Lombroso. Il ne sera pas énoncé ensuite «le » modèle épistémologique qui permettrait de positionner définitivement Lombroso dans l'histoire de sa discipline mais plus prosaïquement deux concepts qui voudraient inciter à une approche plus ouverte de l'histoire des sciences de l'homme.

\section{I. - L'ANTHROPOLOGIE CRIMINELLE DE LOMBROSO ET SON HISTORIOGRAPHIE}

Cesare Lombroso était comme la grande majorité des anthropologues du $\mathrm{XIX}^{\mathrm{e}}$ siècle médecin de formation. Il obtint sa thèse de médecine à Pavie en 1858 avec une étude sur le crétinisme en Lombardie. Exerçant pendant quelques années la fonction de médecin militaire, Lombroso se spécialisa ensuite dans l'étude de la pellagre et de la folie et s'attacha en particulier à mettre en évidence les caractères qui permettraient de distinguer à coup sûr les fous des criminels. C'est en faisant l'autopsie du brigand Vilella qu'il fit sa première «découverte ». Les anomalies anatomiques du cadavre ne pouvaient s'expliquer que si l'on faisait l'hypothèse que « les caractères des hommes primitifs et des animaux inférieurs devaient se reproduire de nos temps ${ }^{3}$ ». Dès lors, Lombroso consacra la plus grande partie de son activité scientifique à l'anthropologie du criminel et il consigna les premiers résultats de ses travaux dans le livre qui allait le rendre mondialement célèbre. Si la première édition en 1876 de Uomo delinquente ne faisait que reprendre deux articles déjà parus, les éditions suivantes furent autant d'occasions pour l'auteur d'ajouter de nouvelles observations, préciser sa pensée et répondre à ses contradicteurs. Dans cette tâche, Lombroso ne travailla pas seul et il fut aidé et stimulé par les travaux plus ou moins distants ou complémentaires de nombre de ses compatriotes parmi lesquels il faut mentionner Enrico Ferri (1856-1929) qui inventa le terme de «criminel-né » et Raffaele Garofalo (1852-1934) qui popularisa celui de « criminologie ».

\section{a) L'homme criminel}

Sur le plan théorique, Lombroso reste célèbre pour avoir tenté d'expliquer le comportement d'un nombre important de criminels par un retour à un type «sauvage » chez lequel le crime serait la norme. L'anthropologue se fondait sur le principe de la récapitulation: tous les enfants des pays

3. Lombroso, 1908, p. XXII. 
civilisés manifestaient des propensions criminelles, comme les primitifs, mais une éducation normale les rendait généralement honnêtes, adaptés à leur société. Lombroso pensait que l'atavisme faisait partager aux infracteurs de nombreuses caractéristiques avec les peuples primitifs qui étaient restés à un stade infantile de l'évolution. Cette identité lui permettait de postuler l'existence d'un véritable type anthropologique spécifique aux criminels-nés qui renvoyait à une étape d'évolution antérieure à la différenciation raciale. L'anthropologue s'attacha essentiellement à recenser les critères anatomiques permettant de déceler un individu atavique mais il ne négligea pas pour autant les critères « sociologiques », comme l'argot et les tatouages, et les critères «physiologiques », comme cette fantasmatique insensibilité à la douleur qui fut à une autre époque la marque de reconnaissance du diable...

Pour appuyer sa théorie de l'atavisme criminel, Lombroso fit une enquête - purement bibliographique - qui confirmait l'origine animale du crime. Le premier chapitre de L'Homme criminel était ainsi consacré à ce que l'auteur appelait, d'une manière très révélatrice, « l'embryologie du crime » (« embryogenèse » convenait mieux). On apprend à sa lecture que les plantes insectivores «commettent de véritables meurtres sur les insectes » et que les animaux sont bien plus criminels encore : les rats, les souris, les brochets, les loups sont cannibales, comme les marsouins, les lapins et les serpents. Les renards sont rusés certes, mais aussi cannibales et parricides. Les singes sont spécialistes du « vol habile et par association », les chats pratiquent le vol domestique (comme la pie) tandis que les fourmis rouges n'hésitent pas à accomplir des "enlèvements de mineurs " ${ }^{4}$. Enfin, le comportement des « chevaux à nez busqué », reconnaissables à leur « front étroit et fuyant » (comme les lièvres) s'avérait très semblable à celui des criminels-nés ayant des anomalies crâniennes. La psychologie chevaline confirmait ces similitudes :

«Ainsi, parmi les chevaux de troupe, on en trouve quelques-uns, rebelles à la discipline, qui font le contraire de ce qu'on leur demande, et conservent un souvenir très vif des mauvais traitements [...] Ils ont beaucoup d'intelligence, mais ils en abusent pour soustraire à leurs compagnons leur portion: Quelquesuns sont traîtres et ne laissent échapper aucune occasion de faire du mal à l'homme ou à leurs compagnons, sans la moindre provocation $[. . .]^{5}$.»

Lombroso retrouvait ainsi chez les animaux toutes les catégories qu'il avait repérées chez les hommes : délits par amour, cupidité ou haine, escroqueries, vols, etc. Cette histoire naturelle du crime se poursuivait avec le

4. Lombroso, 1887 , p. 2-6.

5. Lombroso, 1887, p. 9. 
rappel des mœurs des sauvages et des premières civilisations. L'insensibilité à la douleur évoquée plus haut était pour Lombroso une des caractéristiques que partageaient les criminels-nés et les sauvages car tous les voyageurs connaissaient bien "l'indifférence des nègres et des sauvages d'Amérique à l'égard de la douleur: les premiers se coupent la main en riant, pour échapper au travail; les seconds, liés au poteau de torture, chantent gaiement les louanges de leur tribu, pendant qu'on les brûle à petit feu ${ }^{6}$ ».

Lombroso trouvait dans l'atavisme un argument pour rejeter les objections de ceux qui, à l'instar du juriste Gabriel Tarde (1843-1904), lui rappelaient que la définition du crime variait dans le temps ${ }^{7}$. L'anthropologue italien répondait en effet à ses détracteurs que l'atavisme rendait parfaitement compte de ces variations car, comme toute maladie, il s'exprimait avec différentes intensités : un atavisme faible faisait commettre des crimes autorisés à l'époque médiévale, un atavisme plus fort faisait remonter dans le temps et redescendre dans l'échelle de moralité pour atteindre les crimes autorisés chez les animaux. Ainsi, Lombroso expliquait facilement cette pédérastie et cet infanticide légitime que Tarde tenta de lui opposer :

« [...] l'on ne se rendrait pas un compte bien exact de la diffusion énorme de la pédérastie, de l'infanticide, crimes pour la perpétration desquels on voit souvent se former de vraies associations, si l'on ne se rappelait pas le temps où à Rome, en Grèce, en Chine, à Tahiti, les faits de ce genre, loin de passer pour des crimes, faisaient quelquefois partie des coutumes nationales. [...] Il y a dans l'hérédité une vraie stratification; c'est celle-ci qui favorise, chez nous, la reproduction des instincts de l'homme préhistorique et ceux aussi de l'homme du Moyen Âge. Ainsi s'expliqueraient par exemple les crimes récemment commis par les antisémites, les querelles de clocher, où nous pourrions voir une haine héréditaire datant du Moyen Âge; ainsi que la manie du duel, que rien ne peut réfréner ${ }^{8}$. 》

Considérée sous cet angle, l'explication atavique semblait posséder une certaine cohérence interne. Elle offrait surtout la possibilité de développer des arguments $a d$ hoc à l'infini car tous les crimes autrefois tolérés étaient susceptibles d'être expliqués par cette régression. Lombroso ne convainquit pourtant personne très longtemps avec cette hypothèse car elle se heurta à des résistances de la part de certains psychiatres et anthropologues et il n'expliqua plus, dès le milieu des années 1880 , les « criminels-nés » par le seul atavisme mais aussi par l'épilepsie. C'était ici, comme le notait Lombroso, « la maladie qui venait s'ajouter à la monstruosité ${ }^{9}$ ». Il n'est pas

\footnotetext{
6. Lombroso, 1887, p. 320.

7. TARDE, 1890 .

8. Lombroso, 1887, p. 662-663.

9. LoMBroso, 1887 et 1895 , p. 140.
} 
indispensable pour notre propos d'expliciter les raisons et les linéaments de l'évolution de la théorie lombrosienne. Retenons seulement qu'à terme, Lombroso décrivit les criminels-nés par les phénomènes parfois indépendants, souvent liés, d'atavisme, d'épilepsie et de folie morale. Cet élargissement théorique lui permettait de rendre compte de la manière la plus exhaustive possible de tous les comportements criminels, de l'assassinat au délit politique en passant par le viol ou le vagabondage.

\section{b) La femme criminelle}

Toutes ces observations ne concernaient pourtant que les hommes criminels, mais que devenaient les femmes, dont on savait à l'époque qu'elles commettaient beaucoup moins de délits? Comment l'anthropologie criminelle pouvait-elle expliquer que les femmes soient apparemment moins souvent atteintes d'atavisme et d'épilepsie? Les anthropologues positivistes s'en remirent, comme ils aimaient à le répéter, à la seule observation des « faits » et dans l'ouvrage qu'ils consacrèrent à la femme criminelle, Lombroso et son gendre Guglielmo Ferrero eurent du mal à cacher la satisfaction que leur procurait les résultats :

«Les nouvelles recherches sur la femme criminelle et sur la femme prostituée sont, peut-être, parmi les dernières études de l'anthropologie criminelle, celles qui consacrent le mieux la supériorité de notre méthode d'observation à outrance des faits: unique secret de nos triomphes sur nos adversaires a priori $^{10}$.»

Lombroso et Ferrero avaient tenté de comprendre pourquoi les femmes étaient statistiquement beaucoup moins criminelles que les hommes et à force de patience, de mesures et d'observations accumulées, ils expliquaient l'apparente faiblesse du taux de criminalité féminine en affirmant que la prostitution était l'équivalent «naturel» du crime chez la femme. Les mesures anthropométriques étaient sous cet angle si probantes qu'elles leur avaient donné (enfin!) la clé de la psychologie féminine :

«La coexistence chez la femme, de la cruauté et de la pitié est une autre forme de contradiction pleinement résolue dans nos études par l'influence de la maternité qui, en se greffant sur la cruauté primitive, en fait souvent jaillir la douceur; de même son infériorité en génie, en force et en variabilité, nous explique pourquoi, étant peut-être moins morale, la femme est cependant moins souvent criminelle. Tout cela, joint à l'atavisme et aux puissantes ardeurs masculines, nous aide à comprendre comment l'équivalent de la criminalité innée est chez elle, bien plus que le délit ou le crime, la prostitution, qui

10. Lombroso, Ferrero, 1896, p. I. 
ne devrait cependant pas exister chez un être si peu accessible aux paroxysmes érotiques ${ }^{11}$. »

Il est rare de pouvoir ressaisir en si peu de lignes la fonction d'objectivation d'un discours scientifique, la légitimation d'un imaginaire social et des rapports de force qu'il contient. Loin de voir, comme Victor Hugo, dans les prostituées des esclaves achetés à la misère, les deux anthropologues faisaient la genèse de la prostitution en s'appuyant sur l'observation des « primitifs » et sur les mœurs probables des ancêtres de l'homme civilisé confirmant ainsi le qualificatif de «plus vieux métier du monde » : au début était la prostitution... ${ }^{12}$.

Ces supputations étaient corroborées, d'après Lombroso et Ferrero, par l'abondance de faits d'observation. Dès 1893, par exemple, leurs collègues Ottolenghi et Carrara avaient publié dans les Archives d'anthropologie criminelle lyonnaises un article faisant état d'une recherche de podologie criminelle. L'étude mettait en évidence qu'une anomalie entre le premier et le deuxième orteil était plus fréquente chez les prostituées que chez les femmes normales. Cet écart n'était pas un caractère professionnel comme chez les résiniers, par exemple, mais un authentique caractère atavique. Certes, on pouvait le rencontrer parfois encore chez les "races supérieures" mais il était «bien plus fréquent chez la femme que chez l'homme » ainsi que "chez les dégénérés, et spécialement chez les idiots, criminels, épileptiques et prostituées ${ }^{13}$. Des recherches similaires furent assez fréquentes au sein de l'école italienne. Finalement, Louis Jullien fit une synthèse de ces travaux au $I^{\mathrm{e}}$ congrès international d'Anthropologie criminelle, qui se tint à Genève en 1896. Il démontra en une brillante communication que les faits étaient là : les pieds des prostituées étaient bien souvent préhensiles... comme chez les singes. Bien que représentant un faible nombre d'individus, il existait à côté de ces "prostituées-nées " d'authentiques «criminelles-nées» caractérisables par leurs anomalies physiques. Lombroso fut ici encore très précis sur ces stigmates, allant jusqu'à lier la fréquence de certaines anomalies à des catégories de délits :

"L'empoisonneuse, l'homicide et la voleuse ont le maximum de l'asymétrie crânienne et le maximum de strabisme. Les femmes assassins présentent le maximum de la physionomie virile et mongolique. Les homicides et les empoisonneuses ont un maximum de dépression crânienne, de diastémie des dents, et avec les incendiaires, de nez aplati et difforme. Les homicides, les empoisonneuses et les incendiaires présentent le maximum des zygomes saillants, et,

11. Lombroso, Ferrero, 1896, p. 10-11.

12. Ce scénario éculé fait toujours recette, voir HuL, 1982. Pour une analyse critique plus générale des récits scientifiques de l'anthropogenèse, voir SroczKowski, 1994.

13. Ottolenghi, Carrara, 1893. 
avec les infanticides, le maximum des asymétries faciales et des mâchoires volumineuses ${ }^{14}$.»

Lombroso multipliait sur le plan méthodologique des inductions qui consistaient à rapprocher toutes sortes de «faits » éclectiques, de nature et d'origine souvent fort variées. À cet égard, la consultation de ses œuvres ne peut manquer de dérouter un lecteur contemporain. L'anthropologue mesura les hommes criminels, releva leurs tatouages, leurs écrits, leur langage, leurs antécédents familiaux pour mettre en évidence l'hérédité des penchants, l'influence de la race, du climat, du milieu social, etc. Il appuya ses propositions sur de nombreuses théories mais aussi sur des écrivains, des lois, des coutumes, des faits historiques, des commandements religieux, des croyances populaires et des dictons. Lombroso affubla surtout les criminels d'un grand nombre d'anomalies physiques. Non seulement il recensa les stigmates, mais il assigna à chacun d'eux une origine pathologique. Beaucoup d'anomalies physiques étaient directement imputées à l'atavisme comme « la soudure fréquente de l'atlas avec l'occiput, la saillie des canines, l'aplatissement du palais, la concavité de l'apophyse basilaire, la fréquence de la fosse occipitale moyenne et son développement exagéré (comme chez les lémuriens et les rongeurs); la persistance des poils sur le visage, les arrêts du développement du cerveau, la formation d'un opercule du lobe occipital ${ }^{15}$ ", etc. L'atavisme pouvait se compliquer par l'épilepsie mais aussi par l'imbécillité ou la paralysie générale. Cette liaison entre atar visme et épilepsie, outre qu'elle faisait le pont entre les anthropologues et les aliénistes, autorisait Lombroso à dériver toute une autre série de stigmates physiques de la condition "épileptoïde» des criminels-nés ${ }^{16}$. Notons, enfin, pour ne pas céder à une présentation trop partiale que Lombroso ne refusait pas les facteurs "sociologiques" de la criminalité (misère, mode de gouvernement...) et qu'il reconnaissait à côté des. criminels-nés des criminels par passion, par occasion, et même le «criminellatent » qui, né délinquant, ne se manifeste pas «parce que l'occasion lui manque, ou que la richesse et la puissance lui fournissent les moyens de satisfaire ses mauvais instincts sans enfreindre le code ${ }^{17}$.

\section{c) Lombroso au tribunal de l'histoire}

Comment faire l'histoire de la théorie de Lombroso? Une première possibilité consisterait à calquer la narration historique sur la logique de l'acteur en rendant compte le plus scrupuleusement possible de son argu-

14. Lombroso, Ferrero, 1896, p. 326.

15. LomBroso, 1887 , p. 663.

16. LomBroso, 1887 , p. 657.

17. Lombroso, 1878, 1895, II, p. 589. 
mentation, de ses explications et de son récit de découverte ${ }^{18}$. Si cette façon de construire le récit est la plus simple et la plus spontanée, c'est aussi la moins acceptable dans notre cas puisque plus personne ne croit désormais que la théorie de Lombroso soit vraie. La seconde option qui s'offre à l'historien est en quelque sorte symétrique de la première : elle consiste à organiser le récit en exposant - implicitement et parfois à l'insu même du narrateur, sinon du lecteur - le point de vue des adversaires de Lombroso. Cette forme d'histoire est très bien illustrée dans le récent Médecins et assassins à la Belle Époque, qui réduit la théorie de Lombroso à de fumeuses élucubrations, voire à une "mystification». L'argument essentiel consiste à affirmer que face au "délire positiviste » de l'anthropologue italien, Alexandre Lacassagne (1843-1924) aurait formulé très tôt une "critique sociologique ${ }^{19}$. Cette interprétation fut consacrée $a b$ ovo dans les Archives d'anthropologie criminelle, source partielle et partiale, puisque la revue avait été fondée et animée par Lacassagne qui n'était pas - au sens strict - moins "positiviste " que Lombroso ni beaucoup plus « sociologue ». Une telle lecture est donc le produit lointain d'une historiographie construite à des fins stratégiques de distinction et de positionnement dans le champ scientifique de l'époque ${ }^{20}$. L'épistémologue Patrick Tort a développé sur ce thème une approche plus conceptuelle en reliant la théorie lombrosienne à un conflit d'interprétation qui ne ferait qu'actualiser dans sa structure celui qui eut lieu dans le champ de la tératogenèse entre préformation et épigenèse ${ }^{21}$. Quoique plus nuancée que le récit de Darmon, cette lecture reste prise dans le cadre de la controverse entre l'école française «à dominante sociologique » et la « réduction biologiste pratiquée par les positivistes italiens $"{ }^{22}$. Elle tend ainsi à confondre "l'école italienne » avec l'œuvre de Lombroso, en réduisant cette dernière à sa seule dimension anthropologique.

Ces deux variations narratives partagent, au-delà de leurs différences formelles, une structure d'argumentation qui redouble dans le champ normatif du récit historique l'opération cognitive à laquelle Lombroso se livrait lorsqu'il évaluait, au tribunal ou dans ses recherches, la normalité des individus. Il s'agit toujours peu ou prou, suivant cette analogie juridique, de faire l'histoire de l' "éviction de l'inauthentique par l'authentique $^{23}$ ». Sauf exception, nous sommes tous dans un univers mental qui nous dispose à saisir immédiatement l'extravagance des propos de Lom-

18. Ce récit peut être sujet à interprétation. Voir, par ex., RenNevilLe, 1998.

19. Darmon, 1989.

20. ReNNEVILLE, 1995.

21. TORT, 1989.

22. TORT, 1989 , p. 468

23. Canguilhem, 1988 , p. 33. 
broso. Construire l'histoire de la théorie lombrosienne sur cet effet d'évidence renforce certainement la connivence de l'historien avec ses lecteurs mais elle s'établit au détriment d'une dimension essentielle de l'objet dont il prétend faire l'histoire. Il faut donc mettre autant que possible en suspens nos jugements de valeurs dans notre perception du passé ${ }^{24}$. L'enjeu n'est pas ici de délimiter un lieu objectif d'où l'historien décrirait enfin le réel. Il faut plutôt voir dans cette mise à distance une convergence entre l'écriture historique et celle du roman, défini par Kundera comme un «territoire où le jugement moral est suspendu ${ }^{25} »$. Cette distanciation a d'ailleurs trouvé une expression théorique dans le programme fort de David Bloor sous le terme de "principe de symétrie ${ }^{26}$ ». Ce principe donne une certaine rigueur à nos approches causales - si délicates en histoire - car il incite à considérer avec autant d'exigence la logique de la production de l'erreur qui nous apparaît grossière que celle d'une erreur moins évidente, voire d'une « vérité ».

Le seul préalable à la mise en œuvre de ce principe est d'adopter une définition pragmatique des sciences, qui consiste à ne pas lier le critère de démarcation science/non-science sur le seul état de la science présente mais aussi sur celui de la science passée. Cette définition contingente permet à l'historien des sciences de l'homme de considérer tout à la fois la science dans sa spécificité et son histoire dans sa relativité. La question de la scientificité de Lombroso ne trouve plus alors sa réponse chez les criminologues ou les épistémologues actuels mais chez les savants de l'époque (« savants » définis par leur formation, les références qu'ils mobilisent, leurs lieux de publication et leur participation éventuelle aux congrès scientifiques). Cette méthode exige une mise en contexte de l'œuvre. La carrière scientifique du médecin italien coïncida de fait avec l'âge d'or de l'anthropologie criminelle. Plusieurs revues scientifiques furent créées, en effet, dans les années 1880 pour diffuser cette science en plein essor. Lombroso fonda lui-même l'Archivio di psychiatria, antropologia e scienze penale dès 1880 , son futur rival français Alexandre Lacassagne fonda les Archives d'anthropologie criminelle à Lyon en 1886 et, deux ans plus tard, commença à paraître à Madrid La Revista de antropologia criminal y ciencas medico-legales. Des sociétés savantes furent également créées dans plusieurs pays et le positivisme pénal inspira la création de l'Union internationale de droit pénal en 1889 par Franz Edward von Liszt, Gerard Anton

24. Certeau, 1987 , p. 66-71.

25. KUNDERA, 1992, p. 16.

26. BlOOR, 1982, p. 8. Pour une histoire de ce courant et un état des lieux récent, voir Pestre, 1995. Pour une réception critique de Bloor, voir Isambert, 1985. 
Van Hamel et Adolphe Prins ${ }^{27}$. Enfin, et c'est un fait essentiel dans la vie scientifique de la Belle Époque, l'anthropologie criminelle fit l'objet de sept congrès internationaux de 1885 à 1911 . Force nous est donc de reconnaître que Lombroso représentait un interlocuteur valable pour la communauté scientifique de son époque à la différence par exemple, d'un Gregor Mendel (1822-1884) dont les travaux sur l'hérédité n'attirèrent guère l'attention...

Pour comprendre que ce qui nous apparaît maintenant comme un tissu d'énormités ait pu un jour être confondue avec une théorie scientifique digne d'être discutée, il faut faire intervenir ici ce que l'on propose d'appeler la « rationalité contextuelle » de la théorie de Lombroso. Proche de ce que Georges W. Stocking désignait sous le terme de "reasonableness», cette expression résulte d'une extrapolation de la notion de «subjective rationality » utilisée par Herbert Simon pour décrire la rationalité limitée des sujets et appliquée par Raymond Boudon à l'explication des croyances ${ }^{28}$. La substitution de la « rationalité contextuelle » à la notion de "rationalité subjective" n'implique pas le rejet de l'approche de Boudon. Elle veut marquer la limite de l'objectif visé ici, qui n'est pas d'ébaucher une théorie de la connaissance mais de séparer nettement, dans le récit historique, la question de la « rationalité » de celle de la « vérité ». Plutôt que de circonscrire la rationalité scientifique à une question de méthode, nous l'élargissons à une question d'histoire tant il est « vrai », comme l'a montré Paul Veyne, que les concepts en histoire sont flous parce que « leurs objets bougent tout le temps ${ }^{29}$. L'intérêt de faire varier ainsi l'évaluation de la rationalité en fonction du temps est d'éluder la plupart des questions posées par les lectures présentistes et de déplacer l'objet de notre récit. Le but de l'opération historique n'est plus, en effet, de dire si Lombroso avait tort ou raison, de démontrer en quoi sa théorie était ou n'était pas scientifique, réfutable ou idéologique. Il s'agit plutôt d'organiser la narration sur un objectif que partageaient des auteurs aussi différents que Michel Foucault, Jacques Roger ou Gérard Simon ou Georges Stocking : reconstituer «le régime de plausibilité » de la théorie ${ }^{30}$.

27. Pour le contexte espagnol, voir Huertas, Martinez-Perez, 1993. Pour une revue des travaux allemands sur l'anthropologie criminelle, voir Bleuler, 1896. Pour les théories biologiques de la criminalité aux États-Unis, voir FinK, 1962.

28. Boudon, 1989, 1990. Sur une application de la « raisonnabilité » de Stocking, voir BlanCKaerT, 1988; Di Brizio, 1995, p. 79.

29. VEYNE, 1979, p. 87-96.

30. FouCault, 1969, 1971; Roger, 1995; Simon, 1991. On peut se reporter, pour l'épistémologie de Stocking, à la récente étude de Di BRIzıo, 1995. 


\section{II. - LA RATIONALITÉ CONTEXTUELLE DE LOMBROSO}

Il faut donc rappeler maintenant, au moins brièvement, combien la théorie de Lombroso était plausible dans le contexte scientifique de l'époque. C'est en replaçant l'épisode Lombroso au cœur du moment naturaliste des sciences humaines dans lequel il s'est développé que l'on comprendra mieux sa cohérence. Si l'on considère les grands thèmes mis en œuvre dans sa théorie, on constate que son auteur ne les renouvela pas et qu'il emprunta beaucoup plus qu'il ne créa. On peut citer parmi les plus importants : le rapport du physique et du moral, le comparatisme homme-animal, l'hérédité du crime, la notion d'atavisme bien sûr et le rapprochement entre le crime et la folie. Donnons quelques repères sur ces thèmes.

\section{a) Les interactions du physique et du moral}

L'anthropologie criminelle de Lombroso était sous-tendue par l'idée que le moral et le physique d'un individu étaient liés. Cette idée héritée de la tradition physiognomonique fit florès au $\mathrm{xIx}^{\mathrm{e}}$ siècle comme en atteste l'usage qu'en fit Balzac dans ses romans, le succès populaire des cabinets de cranioscopie dérivés de la phrénologie et les nombreuses éditions populaires des traités de physiognomonie. La mise en évidence des déterminations réciproques du physique et du moral était à la base du programme de l'Idéologie médicale qui considérait, suivant la célèbre formule de Pierre Jean Georges Cabanis (1757-1808), qu'il fallait bien connaître «l'homme physique pour étudier avec fruit l'homme moral ${ }^{31} »$. Cette approche fit des émules dans la communauté des aliénistes dès le début du XIX ${ }^{e}$ siècle. La série de «monomanes " de la Salpêtrière peinte par Géricault est restée célèbre mais le maître Jean-Étienne-Dominique Esquirol (1772-1840) préparait lui-même un ouvrage avec des dessins de plus de deux cents aliénés lorsque la mort le surprit. Au milieu du siècle, les aliénistes Joseph Guislain (1797-1860), Eugène Billod (1818-1886), Bénédict Augustin Morel (1809-1873), Henri Dagonet (1823-1902) s'intéressèrent eux aussi à la question et Henri Legrand Du Saulle (1830-1886) se prit même à rêver la généralisation de la photographie des patients (curables) dans tous les asiles afin de "fixer la marche des accidents intellectuels ${ }^{32}$ ». Dans le champ de la déviance criminelle, l'aliéniste Henry Maudsley (1835-1918)

31. Cabanis, 1805 , t. I, p. 491.

32. Legrand Du Saulle, 1863, p. 258. 
était, par exemple, convaincu que la laideur morale du criminel avait sa correspondance physique :

« [...] c'est encore un autre fait d'observation, que la classe criminelle constitue une variété dégénérée ou morbide de l'espèce humaine, marquée par des caractères particuliers d'infériorité physique et mentale. Cette sorte d'individu, a-t-on justement dit, est aussi distinctement reconnaissable de la classe des ouvriers honnêtes et bien nés qu' "un mouton à tête noire l'est de toutes les autres races de moutons". Aussi un agent de la sûreté ou un directeur de prisons, pour peu qu'il ait quelque expérience, les désigne-t-il sans difficulté dans la foule la plus mêlée, à l'église ou au marché ${ }^{33}$.»

Ces « moutons à têtes noires » étaient facilement repérés et, s'appuyant sur l'expérience de son collègue Bruce Thomson (médecin de la prison générale d'Écosse), Maudsley donnait les signes suivants, dont on conviendra qu'ils sont sans ambiguité :

"Un air de famille les dénonce comme compagnons "marqués, notés et signalés par la main de la nature pour l'œuvre de honte". Scrofuleux, souvent difformes, la tête anguleuse et mal conformée, ils sont stupides, fainéants, rechignés, dénués d'énergie vitale et souvent épileptiques. En général, leur intelligence est médiocre et défectueuse, bien qu'ils soient souvent excessivement rusés, et beaucoup d'entre eux sont faibles d'esprit ou imbéciles ${ }^{34}$. »

Même les adversaires scientifiques de Lombroso partageaient l'espoir de fonder une morphopsychologie scientifique. Tarde, par exemple, qui rejeta la conception atavique tant physique que morale du criminel, ne refusait pas complètement l'idée qu'il puisse exister un «type professionnel » de criminel. L'acuité d'un regard bien exercé avait la force de l'évidence et Tarde répondait ainsi aux fâcheux qui lui opposaient la variabilité des physionomies individuelles :

« [...] pourquoi n'existerait-il pas certaines conformations, plus ou moins caractérisées du reste, propres à révéler les propensions criminelles, quand on sait qu'au premier coup d'œil jeté sur une femme, un homme expérimenté devine infailliblement ses habitudes de prostitution, et qu'avec plus de sûreté encore, paraît-il, les pédérastes se reconnaissent entre eux à travers toutes les classes sociales ${ }^{35}$ ?

Si le magistrat de Sarlat refusait nettement les conclusions des études craniologiques de Lombroso qu'il mettait en contradiction avec d'autres

33. MaudsLey, 1877 , p. $27-28$.

34. MaudsLey, 1877 , p. $28-29$.

35. TARDE, 1888 , p. 522. 
recherches, il tenait pour acquis que les criminels avaient une longueur de bras qui les rapprochait des « quadrumanes ${ }^{36}$ ». Le premier collaborateur de Tarde, et semble-t-il unique, fut le docteur Armand Corre (1841-1908) qui travailla sur l'anthropométrie des criminels au début des années 1880 . S'il fut lui aussi très critique envers Lombroso, son jugement sur les criminels ressemblait toutefois étrangement, subtilités techniques mises à part, à celui de son homologue transalpin. Il lui semblait alors évident que : «Rétrograde atavique ou enrayé infantile, type non dégrossi de la race, anormal par vice tératologique, morbide ou dégénératif, le criminel est un être d'infériorité ${ }^{37} »$.

\section{b) L'embryologie du sens moral}

L'embryologie du crime proposée par Lombroso ne peut manquer de faire sourire les lecteurs contemporains, mais il faut là encore la replacer dans son contexte temporel. Dès 1848, le philosophe Ernest Renan (18231892) songeait plus largement à une « embryogénie de l'esprit humain », qui se fondait sur une analogie avec les sciences naturelles:

«De même qu'à côté de la science des organes et de leur opération, il y en a une autre qui embrasse l'histoire de leur formation et de leur développement, de même à côté de la psychologie qui décrit et classifie les phénomènes et les fonctions de l'âme, il y aurait une embryogénie de l'esprit humain, qui étudierait l'apparition et le premier exercice de ces facultés dont l'action, maintenant si régularisée, nous fait presque oublier qu'elles n'ont d'abord été que rudimentaires ${ }^{38}$. "

Le philosophe disposait pour élaborer sa science d'un moyen simple, car il était évident pour lui que « les phénomènes de l'enfance représentent les phénomènes de l'homme primitif ». Un tel principe comparatif était partagé par beaucoup de savants. Les évolutionnistes darwiniens comme Thomas Huxley (1825-1895) avaient préparé l'horizon théorique de la fin du siècle en posant l'homme en continuité avec les singes. Ce que le naturaliste Ernst Haeckel (1834-1919) baptisait la « loi d'Huxley » supposait, par exemple, que les différences anatomiques entre l'organisation humaine et celle des singes supérieurs étaient beaucoup plus faibles que les même différences entre les singes supérieurs et les singes inférieurs. Haeckel luimême avait énoncé une loi «biogénétique » plus connue sous le terme de théorie de la récapitulation et qui stipulait que l'ontogenèse était une

36. TARDE, 1890 , p. 11.

37. CORRE, 1889 , p. 393

38. Renan, 1948, p. 859. Cet extrait est paru initialement dans De l'origine du langage (1848). Voir aussi PETIT, 1995. 
« récapitulation brève et rapide de la phylogénie [...] Dans cette seconde évolution, l'individu reproduit les plus importantes des métamorphoses que ses ancêtres ont subies ${ }^{39}$ ». C'est ainsi qu'il n'était pas difficile pour ces hommes d'imaginer un type intermédiaire entre les derniers des hommes et les premiers singes anthropoïdes. Les cheveux des enfants n'étaient-ils pas comparables, comme le notait Carl Vogt (1817-1895), au duvet des poussins? Et sous cet angle comparatiste, le «Nègre » ne tendait-il pas «sa main aux singes anthropoïdes ${ }^{40}$ ?

Les parallèles entre les capacités mentales et intellectuelles respectives des animaux et des différentes races d'hommes étaient également très fréquents au $\mathrm{XIX}^{\mathrm{e}}$ siècle et cette "psychologie comparative " fut l'une des sciences reines du moment naturaliste des sciences humaines. D'un côté, on était prêt à reconnaître une lueur d'intelligence dans le mouvement des infusoires et l'origine du sens moral dans les sociétés animales à coopération dense (fourmis, abeilles); de l'autre, on déniait aux peuples primitifs cette complexité en arguant de l'archaïsme de leur structure sociale ${ }^{41}$. Charles Letourneau (1831-1902) affirmait dans ses leçons dispensées à l'école d'anthropologie de Paris qu'il n'existait pas de justice chez les hyménoptères sociaux tout simplement parce qu'il n'y avait point de criminels chez eux. L'absence de déviance s'expliquait par le parfait altruisme des fournis et des abeilles : «Des êtres si absolument adaptés à leur vie sociale que, tous, sans exception, paraissent prêts à se sacrifier, sans hésitation et toujours, pour le bien de la communauté, n'ont évidemment que faire de juridiction et de pénalité ${ }^{42}$. $\gg$ Cette entreprise de dénégation de la valeur morale du "primitif » au profit des insectes sociaux fut poussée si loin qu'elle n'allait pas tarder à fissurer le dogme évolutionniste sur lequel elle se fondait. Le naturalisme anthropomorphique d'Alfred Espinas (18441922) prépara en France ce tournant, car, pour le philosophe français comme pour son homologue anglo-saxon John Lubbock (1834-1913), les sociétés de fourmis étaient, du point de vue de l'organisation sociale, beaucoup plus proche de l'homme que celles des singes... Le constat d'infériorité morale des «sauvages » forma en tout cas un lieu commun des représentations scientifiques (et sociales) de l'époque. Tarde fut, à cet égard, l'un des rares spécialistes d'anthropologie criminelle à la mettre en doute avant la vague critique du courant durkheimien, mais son ami Lacassagne estimait au début des années 1880 qu'il fallait rapprocher le comportement

39. HAECKEL, 1877 , p. 1

40. VoGT, 1865 , p. 618.

41. Sur l'intelligence dans les réactions d'évitements des infusoires, voir RoMANEs, 1887, p. 16. Sur l'apparition du sens moral dans les sociétés animales à forte coopération, voir EsPINAS, 1877.

42. Letourneau, 1891, p. 13. 
des criminels de celui des « hommes sauvages ${ }^{43}$ ». Pour Abel Hovelacque (1843-1896), le vol était un « acte moral » chez certains peuples, comme l'assassinat ${ }^{44}$. Eugène Dally (1833-1887), dans la longue préface qu'il fit au livre de Thomas Huxley, prenait en exemple les Fuégiens, Buchmens, Australiens, Tasmaniens en arguant que ce ne serait qu'un «jeu d'oisif» de prendre la peine de prouver que « sur bien des points, certains animaux sont plus développés que ces peuplades, mentalement et moralement ${ }^{45}$. Des hommes aussi estimés de nos jours que Charles Darwin (1809-1882) ou Marcelin Berthelot (1827-1909) étaient imprégnés de cette pensée hiérarchisante. Le premier estimait que les sauvages possédaient des « règles morales inférieures » et le second surenchérissait en notant, au tournant de notre siècle, que « l'étude des races demeurées sauvages a montré combien leur moralité spéciale était voisine de celle des espèces animales sociables, sinon inférieures pour quelques-unes ${ }^{46}$.

\section{c) Hérédité et atavisme}

L'hérédité du crime était un postulat important dans la théorie de Lombroso. Le thème s'est évidemment renouvelé au $\mathrm{xx}^{\mathrm{e}}$ siècle grâce à la génétique, mais il était déjà exploité à l'époque. Il est vrai que l'on mélangeait alors toutes les formes d'hérédité possibles (Mendel eut une notoriété posthume). L'hérédité des maladies nerveuses et la transmission aux descendants des accidents nerveux semblait un fait acquis grâce aux travaux de Charles-Édouard Brown-Séquard (1817-1894) ${ }^{47}$. S'il y eut bien sûr des savants pour refuser « l'innéité criminelle », beaucoup d'autres n'hésitèrent pas à affirmer l'existence de celle-ci. Le facteur héréditaire avait été évoqué depuis le début du $\mathrm{XIX}^{\mathrm{e}}$ siècle dans l'étiologie du suicide, de la folie et du crime par les plus grands médecins-légistes (François-Emmanuel Fodéré, Mathieu Joseph Bonaventure Orfila...) et aliénistes (Jean-Étienne Dominique Esquirol, Jean-Pierre Falret, Jean-Baptiste Cazauvieilh, Prosper Despine, etc.). Le rapport de l'hérédité au crime fut encore largement défendu à la fin du siècle; et pas seulement dans l'épopée naturaliste des Rougon-Macquart de Zola. En France, par exemple, Théodule Ribot (18391916), qui dirigea la fameuse Revue philosophique et fut professeur de psychologie expérimentale à la Sorbonne puis au Collège de France, croyait fermement à l'hérédité des mauvais penchants :

«On peut appliquer à l'instinct de l'assassinat tout ce qui a été dit du vol. Les cas de transmission héréditaire sont aussi concluants et aussi nombreux. Nous

43. Lacassagne, 1882, p. 215 ; Fauvelle, 1885 ; Vianna, 1887.

44. Hovelacque, 1881 , p. 298-299.

45. Eugène Dally, in HuXley, 1868 , p. 86.

46. DarWIN, 1981, t. I, p. 115-137; Berthelot, 1897, p. 25.

47. Brown-SÉQUARD, 1882. 
avons vu plus haut l'hérédité de l'homicide s'ajouter chez une partie de la famille à l'hérédité du vol, et nous croyons inutile de citer des faits qu'on trouve partout. Le goût inné et incurable du vagabondage, dont les races inférieures et les bohémiens offrent de si beaux exemples, est encore une conséquence incontestable de l'hérédité ${ }^{48}$. »

Rappelons ici, afin d'éviter tout malentendu, un glissement sémantique important entre le $\mathrm{XIX}^{\mathrm{e}}$ siècle et le nôtre sur cette question de l'hérédité : à l'époque, "hérédité " n'est pas encore synonyme d' «innéité » comme c'est le cas aujourd'hui. La pensée hygiéniste et le néo-lamarckisme, fortement implantés dans la pensée médicale en France, permettaient de défendre l'hérédité des caractères acquis, surtout pour les accidents du système nerveux. Ce fait important explique ainsi que la majorité des savants français de la Belle Époque ait pu s'opposer farouchement à l'atavisme lombrosien tout en reconnaissant l'hérédité criminelle par le biais de la théorie de la dégénérescence de Morel. Letourneau, qui préfaça l'édition française de L'Homme criminel, formula sur cette même question de l'hérédité criminelle un constat sans équivoque :

« Nos pénitenciers, nos prisons et nos asiles renferment un bon nombre de ces rejetons des âges antérieurs, chez qui l'hérédité vicieuse est incontestable. Les penchants innés et irréfrénables au vol, au meurtre, au viol, à l'incendie, à l'ivrognerie sont loin d'être rares dans nos sociétés dites civilisées et leur transmissibilité héréditaire est hors de doute ${ }^{49}$."

Darwin lui-même admettait l'influence de l'hérédité dans les cas de vols répétés par les membres de familles aisées (c'est-à-dire : sans « raison»). En outre, il n'excluait pas non plus la possibilité que les « mauvaises dispositions » qui reparaissent « sans cause explicable » dans les familles soient des cas de « retour à l'état sauvage ${ }^{50}$.

La notion d'atavisme, cruciale pour Lombroso parce qu'elle lui valut bien des déboires en France, avait déjà acquis une certaine légitimité dans les sciences. Le concept avait été emprunté à la zootechnie et Darwin, là encore, s'en servit pour justifier certains points de sa théorie de l'hérédité $^{51}$. Dans son ouvrage sur la variation des animaux et des plantes, Darwin fit appel en particulier au phénomène d'atavisme pour illustrer le phénomène de la "panspermie » à l'aide d'une image que n'aurait certainement pas reniée Lombroso :

«Le germe fécondé d'un animal supérieur [...] est bourré de caractères invisibles, propres aux deux sexes, aux deux côtés du corps, et à une longue lignée

48. Rівот, 1882 , p. 99-100.

49. LeTOURNEAU, 1887, p. 66.

50. Darwin, 1981, t. I, p. 133, 149.

51. DARWIN, 1981, p. 36-42. 
d'ancêtres mâles et femelles éloignés de nous par des milliers de générations, caractères qui, comme ceux que l'on trace sur le papier avec une encre sympathique, sont toujours prêts à être évoqués, sous l'influence de certaines conditions connues ou inconnues ${ }^{52}$.»

Le naturaliste Carl Vogt avait d'ailleurs évoqué explicitement l'atavisme dans l'étiologie de la microcéphalie. Les microcéphales, « idiots de naissance », possédaient selon Vogt un mélange de caractères humains et simiesques qui représentait un stade intermédiaire, autrefois normal, mais désormais révolu de l'évolution humaine ${ }^{53}$. Le docteur John L. H. Down (1828-1896) proposa même au milieu des années 1860 une classification « ethnique » des idiots qui se basait sur le fait que $10 \%$ des cas d'idiots reproduisaient le type ethnique du Mongol, quelle que soit la race d'origine de l'idiot. C'était là pour Down un fait qui prouvait qu'il n'existait pas de frontière infranchissable entre les races humaines et que la maladie était capable de détruire la «barrière des races ${ }^{54}$ ». La thèse est passée d'une certaine manière dans les représentations sociales puisque le langage commun préfère aujourd'hui encore le terme de «mongolien » à celui de « syndrome de Down » pour désigner les individus atteints de trisomie $21 \ldots$

\section{d) Le crime et la folie}

Notons enfin que le rapprochement du crime et de la folie effectué par Lombroso ne relevait pas non plus de la corrélation gratuite ou farfelue. Pour une raison pratique d'abord : la gestion des aliénés et des criminels relevait au $\mathrm{XIX}^{\mathrm{e}}$ siècle d'un objectif commun d'ordre public, comme l'atteste bien l'histoire parallèle des asiles et des prisons. Pour une raison théorique ensuite : cette question des rapports entre les aliénés et les infracteurs hantait la médecine légale et le discours des aliénistes depuis - là encore - le début du XIx ${ }^{\mathrm{e}}$ siècle. La Gazette des tribunaux (1825) et les Annales d'hygiène publique et de médecine légale (1829) puis les Annales médico-psychologiques (1843) mais aussi de nombreuses revues médicales se firent l'écho de procès en cour d'assises. Ces causes criminelles concernaient des actes particulièrement horribles (affaires Antoine Léger en 1824, Louis-Auguste Papavoine en 1825, Henriette Cornier en 1826, etc.) dont l'absence apparente de motifs mettait en cause les fondements de la philosophie pénale héritée de la Révolution. Les figures de la folie et du crime ont marqué très vite deux failles dans le sujet de Raison qui préside à la légitimité des États fondés sur la fiction du contrat social. L'ordre et la norme sociale ont été ainsi constamment mis à l'épreuve dans ces deux

52. DARWIN, 1868, t. II, p. 64-65.

53. VoGT, 1867 , p. $199-200$.

54. Down, 1887, p. 217. 
formes de déviance et malgré la distinction originelle de Philippe Pinel (1746-1826), qui désigne symboliquement la fin de l'hôpital général et l'essor à venir des asiles d'aliénés, les représentations du fou et du criminel n'ont cessé au $\mathrm{XIX}^{\mathrm{e}}$ siècle de se recouvrir partiellement. Les aliénistes n'eurent d'ailleurs de cesse de justifier théoriquement la liaison des deux phénomènes avec les concepts hybrides de «monomanie homicide », de « folie morale », de «maladie de la volonté » et de « dégénérescence ». La monomanie homicide - qui se démontrait par une totale absence de sens moral - correspondait à peu près à la « folie morale » que Lombroso avait repris de Henry Maudsley et de James Cowles Prichard (1786-1848). Elle marquait selon l'anthropologue italien le «crétinisme » des criminels-nés au point de vue moral $^{55}$...

\section{III. - APPLICATION SOCIALE ET PRÉSUPPOSÉ COGNITIF}

On pourrait continuer ainsi en montrant ce que le terme d' 'épilepsie» recouvrait pour Lombroso et ses contemporains et combien le rapprochement entre épilepsie et criminalité était crédible, mais les quelques repères posés ci-dessus permettent de constater que Lombroso s'appuyait suffisamment sur les sciences de son temps pour que sa théorie paraisse plausible. Mises bout à bout, les références citées par Lombroso tissent donc un réseau discursif qui rend sa théorie beaucoup plus cohérente. Tort a pu objecter que ces références - en particulier celles qui renvoyaient à Darwin - étaient pour une bonne part illégitimes, mais l'essentiel dans notre démarche n'est pas, rappelons-le, d'évaluer la valeur épistémologique des thèses lombrosiennes ${ }^{56}$. Il n'est pas indifférent en revanche, que les Français aient cru que Lombroso s'appuyait réellement sur Darwin et qu'ils aient vu dans cette référence une raison supplémentaire de refuser l'atavisme criminel. On concédera volontiers que Lombroso suscita des polémiques, que ses méthodes et ses conclusions furent critiquées, mais il ne vint pas à l'idée de ses contemporains d'escamoter ses propos sous prétexte qu'ils n'étaient pas scientifiques. Bien sûr, la plupart de ses adversaires n'hésitèrent pas à réduire sa théorie à une caricature - ce qui facilitait les critiques - mais c'est là un procédé trop fréquent dans les controverses

55. Sur la notion de « folie morale », voir Coffin, 1995. Pour une approche historique des rapports entre la nosographie psychiatrique et les catégories juridiques, voir l'analyse de l'affaire Pierre Rivière in FoucAuLt, 1973, ou les réflexions récentes publiées par Grivors, 1990.

56. TORT, 1989, p. 504-507. 
dites « scientifiques » pour qu'on le considère comme révélateur. Lombroso fut très discuté mais reconnu socialement (il fut nommé commandeur de la légion d'honneur en 1906...) et sa théorie du criminel-né domina la plupart des débats des congrès d'anthropologie criminelle.

\section{a) La dimension sociale de la théorie lombrosienne}

Il reste à comprendre pourquoi la théorie du criminel-né se diffusa si rapidement. Il faut faire jouer ici au moins deux critères : la congruence avec ce que l'on pourrait appeler, à la suite de Lucien Febvre, «l'outillage mental » disponible à la fin du siècle dernier dans le champ scientifique et l'utilité sociale présumée de la théorie. Le premier critère vient d'être développé. Évoquons rapidement le second. Si la société moderne s'était contentée d'un savoir explicatif pour la question criminelle, elle aurait pu continuer à invoquer pour le passage à l'acte l'influence diabolique ou, comme le faisait encore Beccaria à la fin du Xvirl siècle, celle des passions. Une théorie portant l'espoir de faire reculer le crime a plus de chances d'attirer le public et les élites gouvernementales de nos sociétés qu'une théorie qui ne résoudrait que des problèmes purement cognitifs. Ce que cherchaient les anthropologues du xix ${ }^{e}$ siècle, comme les criminologues d'aujourd'hui, c'était avant tout à réduire le taux de criminalité et la théorie de Lombroso était accompagnée - comme toutes les théories criminologiques qui connurent un minimum de considération - d'un programme de réformes pénales. Contrairement à ce qu'affirme Tort, l'école italienne et son biologisme formel n'impliquait pas mécaniquement l'aggravation des peines ni la défense des « vertus rééducatives » du système cellulaire ${ }^{57}$. La psychologie des foules développée par Scipio Sighele (1868-1913) avait, par exemple, comme visée pratique de plaider dans les procès la demiresponsabilité des sujets ${ }^{58}$. Il s'agissait plus fondamentalement pour «l'école italienne » de réviser l'économie des peines en l'adaptant aux types de criminels, ce qui allait à l'encontre du code pénal Zanardelli adopté par l'État italien en 1889. Lombroso estimait, certes, qu'il fallait cesser de s'illusionner sur les criminels-nés et les fous-criminels : récidivistes incorrigibles, ils devaient subir une détention perpétuelle dans des asiles spéciaux afin d'éviter de nouveaux crimes. Il maintenait même la peine de mort pour «quelques rares individualités criminelles » qui récidiveraient sur leurs gardiens malgré un enfermement à vie, mais il rejetait la peine de déportation pour les criminels-nés et lui préférait la "symbiose » qui consistait à canaliser les mauvais penchants des criminels-nés à des fins socialement utiles : on pouvait ainsi employer utilement les assas-

57. TORT, 1989, p. 527.

58. Sighele, 1901, annexes. 
sins à la guerre ou à la chirurgie. L'anthropologue reconnaissait d'ailleurs que beaucoup de criminels-nés avaient réussi ainsi à rester honnêtes en exerçant des professions adéquates ${ }^{59}$.

Le nombre de délits commis par les criminels d'occasion pouvait être réduit par des mesures que les criminologues contemporains qualifieraient de «situationnelles" comme l'éclairage des lieux mal famés des villes. Lombroso était, en outre, comme beaucoup de ses contemporains très sceptique sur l'efficacité de la prison : il préférait, lors d'une première condamnation, une peine d'amende à une incarcération et ne se faisait guère d'illusion sur la capacité du système carcéral à amender les délinquants. Certaines mesures législatives non répressives pouvaient également contribuer à la lutte contre la criminalité : la légalisation du divorce devait empêcher les empoisonnements conjugaux, un gouvernement « vraiment libéral " éviterait les insurrections et «les vengeances anarchistes» et le mariage des ecclésiastiques ferait quant à lui disparaître la plupart des crimes « contre les bonnes mœurs ». Les « criminaloïdes » dépistés à temps ne devaient pas être éliminés mais ils pouvaient subir un «nourrissonnage moral » (sic), qui consistait à placer les jeunes orphelins assistés dans des familles ${ }^{60}$. L'anthropologue italien mettait ainsi l'accent avec son collègue Enrico Ferri sur des «substitutifs pénaux» qui devaient constituer les bases d'une véritable thérapeutique du crime, fondée en amont de l'appareil judiciaire sur la «prophylaxie » de fléaux tels que l'alcoolisme, la pauvreté ou la mauvaise éducation.

Recherche étiologique et « remèdes » étaient donc intimement liés, et tentaient de répondre directement à une question de contrôle social. Le problème de la récidive et de son augmentation fut, par exemple, au cour du débat sur la politique pénale au $\mathrm{XIX}^{\mathrm{e}}$ siècle. En France, les commentateurs du Compte général de l'administration de la justice criminelle ne cessèrent de revenir sur cette question. De 34900 récidivistes entre 1851 et 1855, on passa à 62211 en 1868. Le taux de récidive, qui concernait surtout la petite délinquance, atteignait ainsi $85 \%$ en France ${ }^{61}$. La réponse politique fut donnée en France avec la loi Waldeck-Rousseau de relégation des multirécidivistes du 27 mai 1885, la même année que la loi sur la libération conditionnelle. Le rapport de cette décision politique avec les théories des médecins anthropologues de l'époque était patent et «l'école sociologique de Lyon» y était favorable. L'utilité sociale fut de fait un argument

59. LomBroso, 1899 , p. 543-545.

60. LomBroso, 1899, p. 294-295.

61. Pour ces débats, on pourra se reporter à la présentation de la réédition du Compte général de l'administration de la justice criminelle, 1989. Sur le contexte sociopolitique et la prise en charge des peurs sociales (liées aux "Apaches », anarchistes...) par la médecine au tournant du siècle en France, voir NYE, 1984, HarRIs, 1989. Sur le contexte italien, voir PICK, 1986. 
souvent invoqué dans la controverse qui opposa Lombroso à ses collègues et bien peu de savants adoptèrent, comme Paul Topinard (1830-1911), la défense d'une science pure qui n'était d'ailleurs pas sans arrière-pensées ${ }^{62}$. La nécessité de cet aspect appliqué des théories était à l'époque si prégnant que les plus rudes adversaires de Lombroso l'accusèrent de prôner une théorie fataliste et par conséquent inutile. Lacassagne par exemple, opposa explicitement à Lombroso une « initiative sociale » dont le contenu différait bien peu, sur le fond, de celle de son collègue transalpin ${ }^{63}$. La théorie de Lacassagne ne connut pourtant qu'une diffusion très limitée car elle s'appuyait sur des postulats néo-phrénologiques qui ne correspondaient plus du tout aux références légitimes des communautés scientifiques auxquelles elle était destinée. Lacassagne n'a ainsi conservé une place dans l'histoire de la criminologie qu'au prix d'une réduction de sa théorie à quelques aphorismes souvent cités hors contexte ${ }^{64}$. L'anthropologue italien avait développé inversement une théorie dont la rationalité contextuelle était satisfaisante pour les normes de son époque et dont l'utilité sociale présumée favorisa la diffusion. Lombroso avait tort, bien sûr, mais pour utiliser les expressions de Michel Foucault, sa théorie n'en était pas moins « dans le vrai » du discours des sciences humaines de l'époque. Lombroso ne produisit à ce titre rien de plus qu'une «erreur disciplinée ${ }^{65}$ ».

Replacée ainsi dans le cadre conceptuel et institutionnel de son époque, la théorie du « criminel-né » apparaît si peu originale que pour employer la terminologie proposée par Thomas Kuhn, on pourrait la qualifier de « science normale ». Lombroso n'inventa rien, mais il fit un réel effort pour synthétiser des connaissances qu'il puisa dans la psychiatrie, la médecine légale, l'anthropologie et la psychologie de l'époque. Lombroso chercha à relier toutes ces disciplines par le recours à une pléthore d'études chiffrées et, même si c'était aussi une manière de répondre à ses critiques, l'anthropologue reconnut lui-même qu'il n'avait « fait que donner un corps un peu plus organique à ces conclusions qui pour ainsi dire, flottaient dans l'air, encore indistinctes ${ }^{66} \gg$. La place occupée par Lombroso dans la science de son temps explique en partie le traitement que la mémoire disciplinaire de la criminologie lui fait subir : en le reconnaissant tout à la fois comme un père fondateur et en lui déniant toute valeur scientifique, elle concentre et décharge sur son cas toute la pensée d'une époque. Que la criminologie se soit ainsi édifiée sur le meurtre du père n'est pas l'un des moindres paradoxes de son histoire. Son effet d'occultation a eu, en tout cas, une

62. BlancKaert, 1995 , p. 80-83.

63. LACASSAGNE, 1886, p. 183.

64. ReNNEVILLE, 1995b.

65. Notion développée in Foucault, 1971.

66. Lombroso, 1908, p. 665. 
fâcheuse conséquence historiographique : celle d'éluder l'analyse des facteurs sociopolitiques qui suscitèrent l'émergence de ses premiers énoncés ${ }^{67}$.

\section{b) Vers une archéologie des présupposés cognitifs}

La dimension temporelle de l'histoire est relative à ses objets et c'est dans la phase de conceptualisation de ces derniers que se joue une grande part de la pertinence de nos analyses. La question du «criminel-né » de Lombroso peut bien apparaître à cet égard comme un objet «classique » d'histoire des sciences mais elle peut également indiquer d'autres pistes et devenir ainsi le prélude à de nouvelles recherches. Il faut renoncer pour cela au cadre épistémologique qui fonde l'histoire des discours scientifiques sur celle des seules théories. L'approche alternative peut se déployer dans deux directions, concomitantes sur certains points. La première est celle d'une analyse contextuelle. Si l'on entreprend, en effet, d'expliquer la rationalité de la théorie de Lombroso par sa mise en relation avec des pratiques discursives qui dépassent largement sa propre théorie, on déplace l'exigence d'explication sur ces nouvelles unités: Quand apparaissentelles? Quelles sont leurs conditions de possibilités d'existence et de diffusion? Comment ont-elles pu être prises dans le champ des savoirs scientifiques? Pour répondre à ces questions, il faudrait orienter l'analyse historique sur les énoncés qui contribuèrent à instituer au $\mathrm{XIx}^{\mathrm{e}}$ siècle un Homo criminalis comme objet de savoir. On voit aisément tout ce que doit une telle perspective à la méthode exposée par Foucault dans L'Achéologie $d u$ savoir, mais une analyse fine et exhaustive des discours sur l'homme criminel nous amènerait à discuter un certain nombre de thèses avancées dans Surveiller et punir. La méthode du premier ouvrage, insistant sur la mise en rapport des « milieux discursifs » et « non discursifs », étant bien connue et la critique du second ayant déjà été conduite, ce n'est pas sur ce niveau d'analyse que l'on insistera ${ }^{68}$.

Il est possible en effet d'initier une nouvelle recherche en décomposant la structure même de la théorie lombrosienne. On s'apercevrait alors qu'elle comprend un « noyau dur », élément que Imre Lakatos considérait comme caractéristique de l'heuristique négative des programmes de recherches. On pourrait aussi évoquer à la place de ce « noyau dur » ce que Gérald Holton désignait sous le terme de «thêmata» ou encore ce que Thomas Kuhn avait rangé sous le terme de «metaphysical part of para-

67. Le statut de Lombroso comme "père fondateur » isolé de la criminologie scientifique fut remis en question dès les années trente dans le domaine américain (cf. LiNDESMITH, Levin, 1937). L'effet d'occultation est de fait moins sensible dans les pays où Lombroso n'a qu'une faible influence directe, comme en Angleterre par exemple. Voir GarLand, 1985, p. 73-112.

68. Perrot, éd., 1980, p. 9-56. 
digm $\gg{ }^{69}$. Bien que ces trois concepts ne se recoupent pas dans leur contenu et qu'ils relèvent de conceptions différentes de l'histoire des sciences, ils désignent tous un espace qui est resté partiellement jusqu'ici en dehors des analyses historiques. Cette zone d'ombre est rarement prise comme cadre d'investigation par les épistémologies classiques d'histoire des sciences car elle est faite de persistances, d'inerties et d'apparentes stagnations qui cadrent mal avec le procès historique du progrès des sciences.

On avancera ici l'hypothèse qu'il est possible de repérer dans l'histoire des sciences humaines des « présupposés cognitifs », qui sont des éléments (conceptuels ou méthodologiques) participant à la structure des théories scientifiques. De tels présupposés cognitifs informent plus qu'ils ne déforment les théories et ils se définissent essentiellement par leur résistance à la réfutation et leur persistance dans les discours, au-delà de l'abandon des théories dont ils ont été le socle cognitif. Ces éléments se trouvent souvent enracinés à un niveau inconscient des acteurs qui les défendent et ils peuvent, à ce titre, se retrouver à l'identique dans deux théories apparemment en opposition. Ce fut le cas, par exemple, en anthropologie criminelle dans la fameuse opposition Lombroso-Lacassagne. Les présupposés cognitifs peuvent à l'inverse être revendiqués comme critère de validation méthodologique. C'est ce que firent par exemple en 1909, l'année même du décès de Lombroso, Georges Hervé (1855-1932) et Georges Papillault (1863-1934) :

«L'acte criminel se distingue profondément de tous les actes qui constituent la vie sociale ambiante et il représente, dans cet ensemble coordonné, une véritable anomalie. On lui trouvera donc une première explication quand on pourra faire la preuve que l'auteur du crime diffère, par ses caractères organiques et ses aptitudes, du reste des individus qui constituent la société, et qu'il représente une anomalie, exactement comme l'acte qui est émané de lui $[\text { sic }]^{70}$. "

Le présupposé cognitif mis en œuvre par Lombroso consistait donc à rechercher un rapport de causalité entre l'organisation des individus et leur attitude vis-à-vis de la loi. Ce présupposé émerge en France dès le début du $\mathrm{XIX}^{\mathrm{e}}$ siècle avec la phrénologie de François-Joseph Gall (1758-1828) et on peut en suivre la trace jusqu'aux recherches contemporaines de certains généticiens ou neurobiologistes ${ }^{71}$. Lombroso l'a mis en œuvre mais il n'en fut ni l'initiateur ni le dernier défenseur. Anecdote significative : en 1835, année de naissance de Lombroso, et à Turin, ville où il allait effectuer une

69. Lakatos, 1994, p. 63-66; Holton, 1982, p. 17-30 et surtout 1981, p. 21-47; KuHN, 1983, postf.

70. Hervé, Papillault, 1909, p. 261.

71. ReNNEVILLE, 1997. 
grande partie de sa carrière scientifique, quelques médecins phrénologistes avaient effectué l'autopsie en séance publique d'un assassin qui avait défrayé la chronique. L'examen médico-légal révéla une «physionomie sinistre, barbe rouge, cheveux durs, épais et rougeâtres », un " œil droit atrophié " et une "lèvre supérieure très grosse", ainsi qu'un "nez aplati $»^{72}$.

Prise sous cet angle infrathéorique, la théorie de Lombroso devient beaucoup moins intéressante pour elle-même que pour ce présupposé qu'elle met à jour. Ce dernier suscite de nouvelles questions : Comment l'historien peut-il expliquer la distribution spatiale de ce présupposé? Comment en comprendre la persistance temporelle au-delà de la réfutation des théories successives qui le diffusent? Il est plusieurs façons d'appréhender ce phénomène qui s'inscrit manifestement dans la longue durée de l'histoire de la « criminologie ». L'une, liée à la distinction « science » et « idéologie», verrait dans cette irréductibilité l'ultime preuve de l'inévitable dimension idéologique du discours criminologique. Une alternative plus sensible à l'histoire intellectuelle consisterait à évoquer, à la suite de Jacques Roger, une « mentalité scientifique » qui charrie des « produits fossilisés de l'histoire intellectuelle ${ }^{73}$. Jacques Le Goff a défendu le caractère à la fois flou et concret de ce concept. Son intérêt est, de prime abord, évident pour l'historien des sciences puisqu'il met l'accent sur ce que Fernand Braudel appelait la «longue durée ». Il faudrait se garder toutefois de réifier la notion par l'évocation en dernière instance d'un inconscient collectif, car cette réduction psychologique aurait pour effet pervers de dépolitiser la question des représentations sociales ${ }^{74}$. Le terme de «mentalité » doit donc être employé ici avec précaution car la tentation est grande de glisser d'un usage purement descriptif à un concept explicatif dont la faiblesse est certaine $^{75}$. En fait, l'histoire d'un "présupposé cognitif » tel qu'il a été défini plus haut ne peut être assimilable au sens strict à celle d'une mentalité puisque suivant la définition large mais parfaitement opératoire qu'en a donnée Le Goff, une mentalité est un "système » formé d'un « ensemble d'idées toutes faites que les individus expriment spontanément dans un milieu humain donné à une certaine époque " ${ }^{76}$. En outre, la dimension temporelle de la "longue durée» ne peut s'assigner comme but en histoire des sciences de mettre en évidence des structures immobiles. Ce « degré zéro » du langage de l'historien est un leurre. Prendre une permanence comme

72. Rolandis, 1835.

73. ROGER, 1993, p. XXVII.

74. Braudel, 1984, p. 51 ; Vovelle, 1992, p. 20-23.

75. LLOYD, 1994.

76. LE GoFf, 1983, p. 408. La notion est développée in LE GoFf, 1986. 
objet d'histoire ne suppose pas que l'on nie son historicité. Le noyau cognitif délimité ici (la pathologisation de la déviance criminelle) n'est donc qu'un référent narratif et un récit qui se construirait autour de cet axe devrait s'articuler sur une chronologie et des périodes spécifiques : la phrénologie de Gall n'est pas l'anthropologie criminelle de Lombroso ni la génétique du crime. Ces formes théoriques méritent d'être distinguées, ne serait-ce que parce qu'elles ont servi des projets politiques différents.

Les présupposés cognitifs peuvent ainsi devenir des objets privilégiés pour relier ces configurations apparemment purement théoriques à des facteurs que Foucault désignait comme «non discursifs ${ }^{77}$ ». Il est vrai qu'entre la dénégation positiviste et la critique marxiste, le chemin d'une approche sociocognitive des discours scientifiques est resté longtemps étroit. Faut-il pour autant l'abandonner? Il revient à chacun de se forger son réseau de références, mais les réflexions convergentes de certaines œuvres - souvent indépendantes - incitent à désenclaver nos travaux de la seule perspective d'érudition. Les analyses de Roland Barthes, par exemple, appellent notre vigilance sur le fait que « la science » est dans nos sociétés le «langage-objet» privilégié pour produire des «mythes». L'intérêt de la réflexion du sémiologue sur cette question consistait à ne pas prendre le terme de mythe dans le sens commun et figé d' «erreur » (qui est un héritage de notre ethnocentrisme occidental) mais dans une définition fonctionnaliste, qui attribuait au mythe la fonction de « fonder une intention historique en nature, une contingence en éternité ${ }^{78}$. Cette approche marque une nouvelle fois ses distances avec une épistémologie qui ne peut se résoudre à considérer sur un même plan les théories qui visent « une réalité » et « toutes les billevesées nées des différents désirs humains et des fantasmes dont ils se comblent $»{ }^{79}$. Certes, les analyses de Barthes traquaient essentiellement l'idéologie « petite bourgeoise » dont la dénonciation n'est plus d'actualité, mais on peut s'approprier la dynamique de recherche des Mythologies. Avec Barthes, en effet, le caractère fantasmatique des discours n'est plus pour l'historien un argument de clôture et il permet d'accéder, pour qui accepte de le manier avec précaution, à un niveau essentiel de l'interprétation de certains énoncés. Les discours structurés autour du présupposé cognitif cerné plus haut ont ainsi une dimension mythique en ce qu'ils ont pour effet de normaliser dans l'imaginaire social (c'est-à-dire: de chacun d'entre nous) des catégories normatives construites par le droit. Ce recouvrement partiel et cette substitution d'un discours scientifique à un discours juridique a pour effet politique de naturaliser le fonctionnement effectif de l'appareil judiciaire, qui est souvent en

77. Foucault, 1969 , p. 212 à 215.

78. Barthes, 1990, p. 229.

79. Gaston-GrangER, 1989, p. 571. 
décalage avec le droit des codes. Le diagnostic d'anormalité biologique est en effet toujours posé sur les mêmes catégories sociales : on n'a jamais vu un médecin ou un biologiste chercher le type anatomique ou le gène du politicien corrompu alors que des théories sur les infériorités biologiques des pauvres ou des « marginaux » n'ont cessé d'être avancées durant la difficile phase d'industrialisation des sociétés occidentales. De plus, la recherche des facteurs biologiques de la criminalité connaît un regain d'intérêt en cette fin de $\mathrm{xx}^{\mathrm{e}}$ siècle ${ }^{80}$. Loin de dévaloriser nos objets d'étude donc, cette dimension mythique des discours scientifiques ouvre au contraire un espace d'interprétation où les stratégies épistémologiques dévoilent leur dimension politique.

La conversion d'objet historique accomplie grâce à la notion de "présupposé cognitif " incite, enfin, à élargir le corpus des sources et à recomposer les contours de l'analyse. Le corpus des sources, d'abord, ne pourrait se limiter à des traces écrites. Il faudrait y ajouter les objets dans lesquels il s'est exprimé. Ce sont souvent des pièces de musées ou de laboratoires aujourd'hui oubliés : instruments de mesure, moulages de cerveaux de crânes et de bustes, croquis et peintures. Cet élargissement des voies de l'enquête historique, qui nous rapproche effectivement des archéologues, amène à examiner non seulement la question de la réception et de la diffusion de la théorie lombrosienne mais aussi celle de la «naissance» de la criminologie. Ainsi, lorsque l'on entreprend de décrire la naissance de la criminologie «scientifique » ou "naturaliste», il est classique de faire commencer celle-ci dans le dernier tiers du XIX siècle avec l'œuvre de Lombroso et, dans les meilleurs cas, l'on établit un lien entre le déterminisme biologique de ce dernier et celui de Gall, tout en reconnaissant que Adolphe Quetelet (1796-1874) fut, pour sa part, une sorte de "précurseur » du courant sociologique. Frédéric Chauvaud exprimait encore récemment ce point de vue en affirmant que, « au début de la Troisième République, la criminologie semble surgir brusquement, déjà armée d'un savoir pratique et théorique ${ }^{81}$ ". Cette naissance de la criminologie fixée sur la fin du $\mathrm{XIX}^{\mathrm{e}}$ siècle ne rend pourtant compte tout au plus que d'un phénomène d'institutionnalisation. Certes, le mot même ne date que de 1885, année de la publication d'un ouvrage de Raffaele Garofalo qui s'intitule précisément La Criminologie, mais il faut prendre garde ici à un glissement de sens qui a vu le terme «criminologie » recouvrir a posteriori l'aire sémantique que celui d'anthropologie criminelle, peu à peu déconsidéré, occupait dans le dernier tiers du $\mathrm{XIX}^{\mathrm{e}}$ siècle $^{82}$. La naissance de la «criminologie » au sens

80. Taylor, 1984; Marsh, Katz, 1985; Wilson, Herrnstein, 1986.

81. Chauvaud, 1991, p. 183.

82. Garofalo, 1888. Cette question de vocabulaire recouvre à l'époque des enjeux de méthode et de champ de compétence bien réels. Voir ReNNEviLLE, 1994, p. 199-206. 
actuel du terme est donc bien à rechercher dans celle de l'anthropologie criminelle mais ce glissement de vocabulaire apparemment anodin déplace la question des origines de plus d'un demi-siècle en amont ${ }^{83}$. C'est probablement Christian Debuyst qui a attiré le premier l'attention sur ce point en notant qu'un certain nombre de disciplines (qu'il qualifie de « périphériques ») avaient pris comme objet d'étude, dès la première moitié du $\mathrm{xIX}^{\mathrm{e}}$ siècle, le «fait délinquant ». Comparant les Annales d'hygiène publique et de médecine légale, créées en 1829, et les Archives d'anthropologie criminelle de Lacassagne, qui commencent à paraître en 1886, il reconnaissait que « les thèmes de recherches présentés dans la revue la plus ancienne annoncent et éclairent les prises de position criminologiques prises cinquante ans plus tard ${ }^{84}$ ». Cette façon nouvelle d'envisager l'histoire de la criminologie est particulièrement fertile en ce qu'elle reconsidère un certain nombre de points de l'historiographie classique. La cohérence des travaux de Lacassagne, par exemple, n'est pleinement perceptible que si l'on rappelle son attachement quasi anachronique à la phrénologie et à l'hygiénisme. La notion même de «dangerosité », dont la paternité est souvent attribuée à l'école positiviste italienne, apparaît en fait comme la théorisation tardive d'un mode de contrôle social mis en œuvre en France par la police communale, les maires et les préfets depuis la Révolution française ${ }^{85}$. Cette perspective de longue durée jette encore une lueur différente sur certaines divergences de vues entre psychiatres et juges sur la question de la folie criminelle. La « bataille du prétoire », loin d'être réductible à un conflit socioprofessionnel, apparaît plutôt comme une opposition structurante et fonctionnelle de notre appareil judiciaire ${ }^{86}$. La tension, forte bientôt de deux siècles d'existence, doit être vue comme le résultat d'un compromis historique entre la philosophie des Lumières qui préside à la codification du droit pénal contemporain et la nouvelle représentation du sujet qui se construit dans les nouveaux savoirs sur l' « homme ». Elle présuppose même dans les visions apparemment antagoniques des médecins et des juristes un certain nombre de réquisits communs ${ }^{87}$.

Ce déplacement incite donc à reprendre la question de la naissance des discours scientifiques sur le criminel et de leurs rapports avec les institutions pénales et la gouvernementalité de nos sociétés contemporaines. Des réponses ne pourront être proposées qu'en faisant jouer des facteurs socio-

83. KaluSZYNSKI, 1988.

84. Debuyst, 1990 , p. 364.

85. VAN de KeRChOVE, 1983.

86. FouCAult, 1981.

87. VAN DE KERChOVE, 1990. 
politiques dont le déploiement s'articulerait à une variété de densités temporelles qui n'aurait plus grand-chose à voir avec le temps événementiel que les historiens des sciences ont longtemps privilégié. Pour rendre pleinement intelligible la rationalité du criminel-né de Lombroso, il faut faire jouer des durées différentes qui permettent d'organiser dans un cadre temporel la «multicontextualisation » prônée par George W. Stocking ${ }^{88}$. En référence à la trilogie braudélienne, on peut poser que la première durée est celle du «temps événementiel». Elle correspond ici aux débats dans les congrès d'anthropologie criminelle, aux rivalités personnelles (Lombroso/Lacassagne...). C'est l'échelle de récit classique qui n'appelle guère de commentaire. La seconde durée s'apparente à ce que Fernand Braudel dénommait la « conjoncture ». Elle démontre, à travers l'analyse des rapports sociaux et des concepts des différentes communautés scientifiques (anthropologues, psychiatres, médecins, juristes), que la question de la réception de Lombroso et de sa diffusion est liée au succès et aux résistances suscités par la théorie darwinienne de l'évolution. C'est à ce niveau conjoncturel que l'on peut comprendre pourquoi la théorie de Lombroso suscita en France une levée de bouclier bien que les médecins anthropologues aient appréhendé la question criminelle avec le même présupposé cognitif. L'ultime temporalité, celle de la longue durée, est encore peu empruntée. C'est pourtant dans ce cadre que la dimension mythique - donc fonctionnelle et politique - de certains énoncés scientifiques apparaît avec le plus d'évidence. Un présupposé cognitif qui se déploie sur une longue durée ne peut guère être réduit à la seule coïncidence d'une succession d'erreurs de raisonnement ou de savants déviants. La théorie de Lombroso s'insère ici dans un temps moins fluide et dans un contexte plus large. Délaissant la catégorie de l'« erreur » qui autorise trop souvent une réduction axiologique, l'analyse historique tendrait alors à rechercher les conditions de possibilité du phénomène sociopolitique de réduction médicale des déviances qui dépasse largement en amont comme en aval la théorie du criminel-né ${ }^{89}$. Elle renverrait pour sa

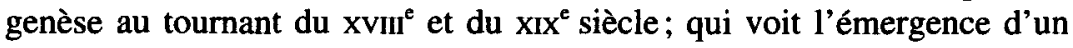
certain nombre de discours sur le vivant dans lesquels le savoir médical occupe une place prépondérante. Elle ferait jouer la construction concomitante d'un nouveau rapport des individus aux institutions, qui prend forme dans un État démocratique basé sur une philosophie contractuelle, sur une nouvelle codification du droit pénal et, à terme, sur un nouveau type de gouvernementalité.

88. Di BRIzıo, 1995, p. 83.

89. ReNNEVILLE, 1997. 
Ces échelles d'analyse ont chacune leur propre légitimité et peuvent être empruntées de manière indépendante. Le concept de « rationalité contextuelle » facilite toutefois le passage de l'une à l'autre et ouvre ainsi la possibilité d'inclure les trois durées dans un récit dont la notion de «présupposé cognitif » serait le fil narratif.

Marc RenNeviLle (avril 1996).

\section{BIBLIOGRAPHIE}

BarThes (Roland), 1990, $1^{\text {re }}$ éd. 1956, "Le mythe aujourd'hui ", in ID., Mythologies, Paris, Seuil, p. 193-247.

BERTHELOT (Marcelin), 1897, Science et morale, Paris, Calmann-Lévy.

Berthelot (M.), 1898, "La vie et les travaux de Brown-Séquard », Revue scientifique, t. IX, 2, p. 800-812.

BLANCKAERT (Claude), 1988, "Story" et "History" de l'ethnologie ", Revue de synthèse, t. CIX, 3-4, p. 451-467.

BLANCKAERT (C.), 1995, « Des sauvages en pays civilisé. L'anthropologie des criminels (1850-1900) », in MucchIELLI, éd., 1995, p. 55-88.

BLEULER (Eugen), 1896, Der geborene Verbrecher. Eine kritische Studie, Munich, J.F. Lehmanns.

Bloor (David), 1982, Sociologie de la logique, Paris, Pandore.

Boudon (Raymond), 1989, «Subjective rationality and the explanation of social behavior ", Rationality and society, t. I, 2, p. 173-196.

Boudon (R.), 1990, L'Art de se persuader des idées douteuses, fragiles ou fausses, Paris, Fayard.

Bourdieu (Pierre), 1995, « La cause de la science. Comment l'histoire sociale des sciences sociales peut servir le progrès de ces sciences", Actes de la recherche en sciences sociales, 106-107, p. 3-10.

Braudel (Fernand), 1984, Écrits sur l'histoire, Paris, Flammarion.

BROWN-SÉQuARD (Charles-Édouard), 1882, «Faits nouveaux établissant l'extrême fréquence de la transmission par l'hérédité d'états organiques morbides, produits accidentellement chez les ascendants », Comptes rendus de l'Académie des sciences, t. 94, p. 697-700.

Cabanis (Pierre-Jean-Georges), 1805, Rapports du physique et du moral de l'homme, 2 vol., Paris, Crapart, Caille et Ravier.

CANGUILHEM (Georges), 1988, Idéologie et rationalité dans l'histoire des sciences de la vie, Paris, Vrin.

CERTEAU (Michel de), 1987, Histoire et psychanalyse entre science et fiction, Paris, Gallimard.

Chauvaud (Frédéric), 1991, De Pierre Rivière à Pierre Landru. La violence apprivoisée au XIX siècle, Paris, Brépols.

CoFfin (Jean-Christophe), 1995, « La "folie morale". Figure pathologique et entité miracle des hypothèses psychiatriques au XIx siècle ", in MuCCHIELl, éd., 1995, p. 89-106. 
Compte général de l'administration de la justice criminelle en France pendant l'année 1880 et rapport relatif aux années 1826 à $1880,1989,1^{\mathrm{re}}$ éd. 1882, prés. par Michelle PERRot et Philippe RoBERT, Paris/Genève, Slatkine Reprints, p. 1-30.

CORRE (Armand), 1889, Les Criminels : caractères physiques et psychologiques, Paris, Douin.

Darmon (Pierre), 1989, Médecins et assassins à la Belle Époque, Paris, Seuil.

DARWIN (Charles R.), 1868, De la variation des animaux et des plantes sous l'action de la domestication, 2 vol., Paris, Reinwald.

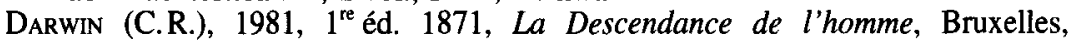
Complexe.

Debuyst (Christian), 1990, «Pour introduire une histoire de la criminologie : les problématiques de départ », Déviance et société, vol. XIV, 4, p. 347-376.

Di BRIzıo (Maria Beatrice), 1995, " "Présentisme" et "historicisme" dans l'historiographie de G.W. Stocking », Gradhiva, 18, p. 77-89.

Down (John Haydon Langdon), 1887, $1^{\mathrm{re}}$ éd. 1866, « Observations of an ethnic classification of idiots ", in ID., On some of the mental affections of childhood and youth [...] together with other papers, Londres, J. \& A. Churchill, p. 259-262.

Espinas (Alfred), 1877, Des sociétés animales. Étude de psychologie comparée, Paris, Germer-Baillière.

Fauvelle (René), 1885, « De la fréquence des crimes et délits chez les inférieurs », L'Homme, t. 2, p. 161-163.

Fink (Arthur E.), 1962, $1^{\text {re }}$ éd. 1938, The Causes of crime. Biological theories in the United States : 1800-1915, New York, Barnes \& Cie.

Foucault (Michel), 1969, L'Archéologie du savoir, Paris, Gallimard.

Foucault (M.), 1971, L'Ordre du discours. Leçon inaugurale au Collège de France, Paris, Gallimard.

Foucault (M.), éd., 1973, Moi, Pierre Rivière, ayant égorgé ma mère, ma saeur et mon frère..., Paris, Julliard.

Foucault (M.), 1975, Surveiller et punir, Paris, Gallimard.

Foucault (M.), 1981, "L'évolution de la notion "d'individu dangereux" dans la psychiatrie légale », Déviance et société, vol. V, 4, p. 403-422.

Garland (David), 1985, Punishment and welfare. A history of penal strategies, Aldershot, Gower.

Garofalo (Raffaele), 1888, $1^{\text {re }}$ éd. 1885, La Criminologie, Paris, Alcan.

GASTON-Granger (Gilles), 1989, «Épistémologie », in Encyclopaedia Universalis, Paris, t. 8, p. 565-572.

Goring (Charles), 1972, $1^{\text {re }}$ éd. 1913, The English Convict. A statistical study, Montclair, NJ, Patterson Smith.

Grivols (Henri), éd., 1990, Les Monomanies instinctives, Paris, Masson.

HAECKEL (Ernst), 1874, Histoire de la création des êtres organisés d'après les lois naturelles, Paris, Reinwald et $C^{\text {ie }}$.

HaECKel (E.), 1877, $1^{\text {re }}$ éd. 1874, Anthropogénie ou histoire de l'évolution humaine, Paris, Reinwald et $\mathrm{C}^{\text {ie }}$.

HARris (Ruth), 1989, Murders and madness. Medecine, law and society in the fin de siècle, Oxford, Clarendon Press.

Hervé (Georges), 1909, "Les Trois Glorieuses de 1859 et leur cinquantenaire ", Revue de l'école d'anthropologie de Paris, t. 19, p. 1-14.

Hervé (Georges), Papillault (Georges), 1909, «Le cerveau de l'assassin Gagny. Étude morphologique », Revue de l'école d'anthropologie de Paris, t. 19, p. 245-262. 
HILl (Kim), 1982, «Hunting and human evolution », Journal of human evolution, t. 11, p. 521-544.

Holton (Gérald), 1981, "Les thêmata dans la pensée scientifique », in ID., L'Imagination scientifique, trad. de l'américain Jean-François RoBerTs, Paris, Gallimard, p. 21-47.

Holton (G.), 1982, $1^{\text {re }}$ éd. 1973, L'Invention scientifique. Thêmata et interprétation, Paris, Presses universitaires de France.

HovelaCQue (Abel), 1881, Les Débuts de l'humanité. L'homme primitif contemporain, Paris, Doin.

Huertas (Rafael), Martinez-Perez (José), 1993, « Disease and crime in Spanish positivist psychiatry », History of psychiatry, t. IV, p. 459-481.

HuxLey (Thomas Henry), 1868, $1^{\mathrm{re}}$ éd. 1863, La Place de l'homme dans la nature, Paris, Baillière.

IsAMBERT (François), 1985, «Un "programme fort" en sociologie de la science? », Revue française de sociologie, t. XXVI, 3, p. 485-508.

KALUSZYNSKI (Martine), 1988, «Aux origines de la criminologie : l'anthropologie criminelle ", Frénésie, t. II, p. 17-30.

Kunn (Thomas S.), 1983, $1^{\text {re }}$ éd. 1962, La Structure des révolutions scientifiques, trad. de l'américain par Laure MeYer, Paris, Flammarion.

Kundera (Milan), 1992, Les Testaments trahis, Paris, Gallimard.

KuRELla (Hans), 1893, Naturgeschichte des Verbrechers, Stuttgart, Verlag von Ferdinand Emke.

LABADIE (Jean-Michel), 1995, « Corps et crime. De Lavater (1775) à Lombroso (1876) », in Histoire des savoirs sur le crime et la peine, éd. C. DeBuYst et al, Bruxelles, De Boeck, p. 295-345.

LACASSAGNE (Alexandre), 1882, « L'homme criminel comparé à l'homme primitif », Bulletin du Lyon médical, p. 210-217, 244-255.

LACASSAGNe (A.), 1886, "Congrès d'anthropologie criminelle de Rome », Archives d'anthropologie criminelle, t. I, p. 86-92, 107-186, 279-286.

LaKatos (Imre), 1994, $1^{\text {re }}$ éd. 1978, Histoire et méthodologie des sciences, trad. de l'anglais Catherine Malamoud, Jean-Fabien SPITZ, sous la dir. de Luce GIARD, Paris, Presses universitaires de France.

LE GoFf (Jacques), 1983, " Histoire des sciences et histoire des mentalités », Revue de synthèse, $\mathrm{t}$. CIV, 111-112, p. 407-415.

Le GoFf (J.), 1986, $1^{\mathrm{re}}$ éd. 1974, «Les mentalités, une histoire ambiguë », in Faire de l'histoire, éd. Jacques Le Goff et Pierre Nora, t. 3, p. 106-129.

Legrand Du SAUlle (Henri), 1863, « De l'application de la photographie à l'étude des maladies mentales (extrait de la séance du 27 avril 1863) ", Annales médico-psychologiques, 2, p. 256-260.

LETOURNEAU (Charles), 1887, L'Évolution de la morale. Leçons professées pendant l'hiver de 1885-1886, Paris, Delahayer \& Lecrosnier.

LETOURNEAU (C.), 1891, L'Évolution juridique dans les diverses races humaines, Paris, Lecrosnier \& Babé.

LindeSmith (Alfred), Levin (Yale), 1937, « The Lombrosian myth in criminology », The American Journal of sociology, t. 42, 5, p. 653-671.

Lloyd (Geoffrey Ernest Richard), 1994, Pour en finir avec les mentalités, trad. de l'anglais Franz ReGNot, Paris, La Découverte.

Lombroso (Cesare), 1878, $1^{\text {re }}$ éd. 1876, L'Uomo delinquente, in rapporto all'antropologia, giurisprudenza e alla discipline carcerale, Turin, Bocca.

Lombroso (C.), 1887 et 1895, L'Homme criminel. Criminel-né, fou moral, épileptique, 2 vol. + atlas, Paris, Félix Alcan. 
Lombroso (Cesare), Ferrero (Guglielmo), 1896, La Femme criminelle et la prostituée, Paris, Félix Alcan.

Lombroso (C.), 1899, Le Crime, causes et remèdes, Paris, Schleicher Frères.

Lombroso (C.), 1908, "Discours d'ouverture ", Actes du sixième congrès international d'Anthropologie criminelle, Turin, Bocca, p. XXI-XXVI.

MARSH (Frank H.), KaTZ (Janet), éd., 1985, Biology, crime and ethics. A study of biological explanations for criminal behavior, Cincinnati, $\mathrm{OH}$, Anderson Publishing.

Maudsley (Henry), 1877, Le Crime et la folie, Paris, Germer et $C^{i e}$.

MuCCHIELl (Laurent), éd., 1995, Histoire de la criminologie en France, Paris, L'Harmattan.

NyE (Robert A.), 1984, Crime, madness and politics in Modern France. The medical concept of National Decline, Princeton, NJ, Princeton University Press.

Ottolenghi (Salvatore), Carrara (Mario), 1893, "Le pied préhensible au point de vue de la médecine légale et de la psychiatrie », Archives d'anthropologie criminelle, t. VIII, p. 480-492.

Perrot (Michelle), éd., 1980, L'Impossible Prison, Paris, Seuil.

PESET (Jose Luis), 1975, Lombroso y la escuela positivista italiana, Madrid, Éditions Castilla.

Pestre (Dominique), 1995, «Pour une histoire sociale et culturelle des sciences. Nouvelles définitions, nouveaux objets, nouvelles pratiques », Annales. Histoire, sciences sociales, 3 , p. 487-522.

PetrT (Annie), 1995, "Étudier "l'embryogénie de l'esprit" ", in Le XIX siècle. Science, politique et tradition, éd. Isabelle PoutrIn, Paris, Berger-Levrault, p. 277-293.

PICK (Daniel), 1986, « The faces of anarchy. Lombroso and the politics of criminal science in post-unification Italy », History Workshop, 21, p. 60-86.

Renan (Ernest), 1948, $1^{\mathrm{re}}$ éd. 1890, L'Avenir de la science. Pensées de 1848, in Euvres complètes, éd. Henriette Psichari, Paris, Calmann-Lévy, vol. 3, p. 7131151.

RenNeVILle (Marc), 1994, «L'anthropologie du criminel en France », Criminologie, t. XXVII, 2, p. 185-209.

RenNeville (M.), 1995a, «La réception de Lombroso en France (1880-1900) », in MuCChIELl, éd., 1995, p. 107-135.

RenneVille (M.), 1995b, «Alexandre Lacassagne. Un médecin-anthropologue face à la criminalité (1843-1924)», Gradhiva, 17, p. 127-140.

ReNNeVILle (M.), 1997, La Médecine du crime. Essai sur l'émergence d'un regard médical sur la criminalité en France. 1785-1885, 2 vol., Lille, ANRT-Presses universitaires du Septentrion.

RENNEVILle (M.), 1998, «Lumière sur un crâne? Une lecture en miroir de la découverte de l'atavisme criminel ", in La Découverte et ses récits, éd. Jacqueline CARroy et Nathalie Richard, Paris, L'Harmattan (à paraître).

RiBot (Théodule), 1882, L'Hérédité psychologique, Paris, Germer Baillière et $C^{\text {ie }}$.

RogER (Jacques), 1993, "L'histoire des sciences : problèmes et pratiques, histoire des sciences, histoire des mentalités, micro-histoire ", in Les Sciences de la vie dans la pensée française du XviIf siècle, préf. de Claire SALOMON-BAYET, $3^{\mathrm{e}}$ éd. Paris, Albin Michel, av.-pr., p. XIX-XXXIX.

ROGer (J.), 1995, Pour une histoire des sciences à part entière, Paris, Albin Michel.

RolandIS (Giuseppe de), 1835, «Lettre à M. le docteur Fossati, sur un criminel convaincu de plusieurs viols, suivis de meurtre », Journal de la Société phrénologique de Paris, avril, p. 244-247.

Romanes (George John), 1884, L'Évolution mentale des animaux, Paris, Reinwald. 
Romanes (G.J.), 1887, L'Intelligence des animaux, Paris, Alcan.

Sighele (Scipio), 1901, $1^{\text {re }}$ éd. italienne 1891, La Foule criminelle. Essai de psychologie collective, Paris, Alcan.

Simon (Gérard), 1991, « De la reconstitution du passé. À propos d'histoire des sciences, entre autres histoires », Le Débat, 66, p. 134-147.

SToczкоwsкi (Wiktor), 1994, Anthropologie naïve, anthropologie savante. De l'origine de l'homme, de l'imagination et des idées reçues, Paris, CNRS-Éditions.

TARDE (Gabriel), 1888, «La criminologie », Revue d'anthropologie, $3^{\mathrm{e}} \mathrm{S}$., t. III, p. 521-533.

TARDE (G.), 1890, La Criminalité comparée, Paris, Alcan.

TAYLOR (Lawrence), 1984, Born to crime. The genetic causes of criminal behavior, Londres, Greenwood Press.

TORT (Patrick), 1989, La Raison classificatoire, Paris, Aubier.

VAN DE KeRCHOve (Michel), 1983, «Le juge et le psychiatre. Évolution de leurs pouvoirs respectifs ", in Fonction de juger et pouvoir judiciaire, éd. Philippe Gérard, Michel Van De Kerchove et François Ost, Bruxelles. Publications des facultés universitaires Saint-Louis, p. 311-390.

VAN DE KeRChOve (M.), 1990, «Droit pénal et santé mentale », Déviance et société, vol. XIV, 2, p. 199-206.

Veyne (Paul), 1979, Comment on écrit l'histoire, suivi de Foucault révolutionne l'histoire, Paris, Seuil.

VIANNA (Arthur), 1887, «L'homme primitif actuel », Revue scientifique, t. 2, p. 621629.

VoGT (Carl), 1865, Leçons sur l'homme. Sa place dans la création et dans l'histoire de la terre, Paris, Reinwald.

VoGt (C.), 1867, Mémoire sur les microcéphales ou hommes-singes, Genève/Bâle, Georg.

Vovelle (Michel), 1992, Idéologies et mentalités, Paris, Gallimard.

WiLson (James Q.), HerRnsteIN (Richard J.), 1986, Crime and human nature. The definitive study of the causes of crime, New York, Simon \& Shuster. 